\title{
Biologia de Abertura e Fechamento do Tubo de Acesso de Nannotrigona testaceicornis (Lepeletier) (Apidae: Meliponini)
}

\author{
Luis Henrique Alves ${ }^{\circledR}$, Leonardo Almeida Sousa ${ }^{2}$, André Felipe Barreto-Lima ${ }^{3}$, \\ Georgina Maria Faria Mucci $^{4} \&$ Fábio Prezoto $^{5}$
}

1. Universidade Severino Sombra, e-mail: lhsalves@hotmail.com (Autor para correspondência ${ }^{\varpi}$ ). 2. Universidade Federal de Viçosa, e-mail: leonardo.a@ufv.br. 3. Universidade de Brasília, e-mail: afblima@hotmail.com. 4. Faculdades Integradas de Cataguases, e-mail: f.mucci@superig.com.br. 5. Universidade Federal de Juiz de Fora, e-mail: fabio.prezoto@ufjf.edu.br.

\section{EntomoBrasilis 8 (1): o8-11 (2015)}

Resumo. O tubo de acesso ao ninho das abelhas sem ferrão está relacionado com a defesa, forrageamento e regulação físico-química, através do controle da temperatura e circulação de ar dentro da colônia. Por isso, sua abertura e fechamento provoca um conflito (Trade-off) sobre o tamanho da entrada do ninho e a segurança da colônia. Este trabalho descreve o processo de abertura e fechamento do tubo de acesso em diferentes períodos do ano, e fornece informações importantes sobre a atividade de forrageamento de Nannotrigona testaceicornis (Lepeletier). Durante o trabalho, conduzido no campus da Universidade Federal de Juiz de Fora (Minas Gerais, Brasil) foram realizadas 41 observações, 27 realizadas no período quente e húmido (de outubro até março) e 14 no período frio e seco (abril até setembro), totalizando 172 horas de observação. Pode-se observar que o tempo gasto durante o fechamento foi significativamente mais longo durante o período quente e úmido $\left(\chi^{2}=7,50 ; p<0,0062\right)$, do que durante $o$ período frio e seco. O tempo de fechamento do tubo mais longo no período quente e úmido, e mais rápido durante o período frio foi regulado pelo ritmo de atividade externa das abelhas, que são influenciadas pelos fatores climáticos. Por isso, o processo de abertura e fechamento sofre alterações durante os diferentes períodos do ano. Desta forma, este trabalho fornece importantes informações sobre a biologia desta espécie, que podem ser empregadas em seu manejo, aumentar sua utilização em atividades comerciais e diminuir a perda de operárias durante a migração das colônias.

Palavras-chave: Abelhas Sem Ferrão; Entrada do Ninho; Tráfego de Forrageamento.

\section{Biology of Opening and Closing the Access tube Nannotrigona testaceicornis (Lepeletier) (Apidae: Meliponini)}

Abstract. The bee nest's access tube relates to its defense, forage and physicochemical regulation through temperature and air circulation control. Therefore, it's opening and closing causes a conflict (Trade-off) in the size of the nest's opening and the colony's safety. This paper describes the process of opening and closing of the access tube in different times of the year and provides important information about the foraging behavior of Nannotrigona testaceicornis (Lepeletier). During this work, conducted in the Universidade Federal de Juiz de Fora campus (Minas Gerais, Brasil), 41 observations were performed, of which 27 took place during the hot and humid season (from October to March) and 14 during the cold and dry season (from April to September), totaling 172 hours of observation. It was seen that the time spent in closing was significantly longer in the hot and humid season $\left(\chi^{2}=7.50 ; p<0.0062\right)$, than in the cold and dry season. The longer closing time of the tube during the hot season and shorter in the cold season was regulated by the bee's external activity rhythm, which is influenced by climatic factors. Thus, the opening and closing processes suffers alterations during the different seasons of the year. Accordingly, this paper shows important information about the species' biology, information that may be used on its handling, increase its use on commercial activities and decrease the loss of workers during colony migration.

Keywords: Foraging Traffic; Nest Entrance; Stingless Bees.

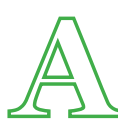
região neotropical possui $75 \%$ das espécies de meliponíneos conhecidos, estes são representados por mais de 30 gêneros, distribuídos em aproximadamente 400 espécies, sendo que mais da metade destas, já foram registradas em território brasileiro (SILvEIRA et al. 2002; CAMARGO \& PEDRO 2003).

Os meliponíneos ou abelhas sem ferrão são insetos eussociais, onde os indivíduos vivem em colônias perenes, ou seja, estão ativas durante todo ano. Estas colônias possuem de centenas até milhares de operárias, algumas centenas de zangões e geralmente uma rainha (MicheNer 2000; Roubik 1989). Elas constituem um grupo de abelhas que não utiliza o ferrão (órgão de defesa) para defesa, pois apresentam o ferrão atrofiado. Por isso, utilizam diferentes estratégias para se defender, entre as mais comuns podemos destacar: a construção dos ninhos em locais de difícil acesso, ataques agressivos seguido de mordidas nas asas, pernas e antenas de um possível intruso, construção de uma entrada, na forma de um tubo de resina, utilização de resina pegajosa no tubo de acesso além de depositar resina pegajosa sobre os invasores
(Nogueira Neto 1997; Michener 2000).

O ninho da maioria das espécies de abelhas sem ferrão apresenta características peculiares. Eles podem ser construídos em cavidades de árvores, no solo ou expostos na parte externa de galhos de árvores ou fendas de rochas. A Estrutura de entrada do ninho (tubo de acesso) varia em forma e material, comumente ela mostra detalhes arquitetônicos característicos, que torna mais fácil a identificação de algumas espécies. Geralmente a entrada é construída de barro associado ou não com cerume ou apenas constituído por um tubo de cerume, cujo comprimento varia entre as espécies (CAMARGo \& WITTMANN 1989). Esta entrada projeta-se a partir da base da cavidade onde se encontra o ninho. Em abelhas que constroem a entrada na forma de um tubo de cerume, a parte distal apresenta diâmetros menores que a largura média do tubo.

A entrada do ninho não está apenas relacionada com a defesa e alimentação, mas também com a regulação físico-química, através do controle da temperatura e circulação de ar que 

das abelhas (BiesmeiJer et al. 2005; Silva et al. 2013). Por isso, algumas espécies fecham a entrada do tubo durante a noite, para apresentar uma maior eficiência contra inimigos naturais e manter internamente a colônia em condições ideias (Wille \& Michener 1973). Como o tubo de acesso é o local de comunicação das abelhas com o ambiente externo, seu diâmetro pode expressar as pressões seletivas ambientais, associadas a ameaças presentes no local onde está o ninho (Couvillon et al. 2008). A abertura e fechamento do tubo provoca um conflito (Trade-off) sobre o tamanho da entrada do ninho e a segurança da colônia. O tubo deve ser grande o suficiente para o tráfego de forrageamento das abelhas, mantendo as condições internas ideais para o desenvolvimento da colônia e ao mesmo tempo deve ter um tamanho que forneça segurança para a colônia e facilite o fechamento durante a noite (WILSON 1971; MichenER 1974; RoubIK 1989).

Entre os Meliponíneos a espécie Nannotrigona testaceicornis (Lepeletier), conhecida popularmente como "Irá́", apresenta comportamento eussocial, possuí colônias numerosas com aproximadamente 25.000 operárias, algumas dezenas de zangões e apenas uma rainha (LindAUER \& KERR 1960; Silveira et al. 2002). Ela destaca-se por possuir grande distribuição, principalmente do sul da região nordeste até o Rio Grande do Sul (Michener 1974; Silveira et al. 2002). Fora do Brasil sua distribuição restringe-se a países localizados na faixa tropical do sul continente americano até o México (Nogueira Neto 1997). Nesta espécie, a entrada do ninho é constituída de um tubo de cerume de cor escura, que durante o dia permanece com muitas abelhas pousadas na parte interna do tubo mantendo vigilância. Durante a noite esta entrada é vedada com uma tela de cerume para proteger o ninho de inimigos naturais e manter estáveis as condições internas do ninho (NogueIRA NeTo 1997). Por apresentar ampla distribuição geográfica, fácil adaptação a ambientes antrópicos e não ser agressiva, $N$. testaceicornis, tem sido muito empregada em estudos de polinização de espécies cultivadas, podendo ser utilizadas também em polinização dentro de estufas, devido à facilidade em manejá-las (NoguEIRA NETO 1997; SlaA 2000).

Mesmo apresentando um grande potencial para ser explorada em atividades comerciais, há poucos trabalhos que abordam os aspectos biológicos desta espécie. A maior parte deste conhecimento é empírico, e encontra-se retido basicamente pelos meliponicultores (criador de abelhas sem ferrão). Portanto, o objetivo deste estudo foi descrever o processo de abertura e fechamento do tubo de acesso em diferentes períodos do ano, além de fornecer informações sobre a atividade de forrageamento desta espécie.

\section{MATERIAL E MÉTODOS}

O estudo foi conduzido no Campus da Universidade Federal de Juiz de Fora, município de Juiz de Fora, Minas Gerais, localizada na região sudeste do Brasil. De acordo com a classificação de KöPPEN (1970), o clima é Cwa - tropical de altitude, com um período quente e úmido de setembro a março e outro período mais frio e seco, de abril a agosto.

Para descrever o processo de abertura e fechamento do tubo de acesso de $N$. testaceicornis foram realizadas 41 observações, 27 realizadas no período quente e úmido (de outubro até março) e 14 no período frio e seco (abril até setembro), totalizando 172 horas de observação em 20 colônias amostradas. Nestas observações foram registrados o comportamento e o número de indivíduos que participaram do processo de abertura e fechamento do tubo de acesso ao ninho. As observações se iniciaram antes de qualquer atividade de voo e foram acompanhados até o termino de todas as atividades das abelhas. Os dados abióticos (temperatura e umidade relativa do ar) foram registrados através do termo higrômetro digitais (Sundo $($ ) ) nas proximidades do tubo de acesso das colônias.

A diferença entre o tempo de abertura e fechamento do tubo de acesso deN.testaceicornis foi realizado pelotestenão-paramétrico de qui-quadrado $\left(\chi^{2}\right)$, utilizando o nível de significância de $95 \%$.

\section{RESULTADOS E DISCUSSÃO}

Para iniciar a abertura do tubo as abelhas operárias cortavam a tela de cerume com suas mandíbulas empurrando-a para dentro ou fora da colônia. Inicialmente 1 ou 2 abelhas começavam a cortar a tela, mas o número de abelhas envolvidas nesta atividade chegou a 22 indivíduos, principalmente no final do processo, quando o diâmetro alcançado pela abertura permitiu o posicionamento de um maior número de indivíduos. O fechamento se iniciou com a diminuição das atividades de forrageamento das abelhas. Nesta etapa as operárias retiravam o cerume do interior da colônia, manipulam com suas mandíbulas e com o primeiro par de pernas o moldavam para construir a tela da entrada do tubo (Figura 1). Para auxiliar o fechamento, as operárias dobravam a borda do tubo reduzindo seu diâmetro. Com a perda da acuidade visual, devido à presença da tela de cerume na entrada do tubo, o registro das atividades comportamentais de fechamento foi diminuindo até serem encerradas por completo. O número médio de abelhas que participaram do fechamento de entrada foi de seis indivíduos, mas pode-se registrar até 26 abelhas, principalmente no inicio do fechamento. O comportamento de fechamento do tubo de acesso é uma estratégia utilizada como defesa em colônias de abelhas Sem Ferrão, pois diminui a ação de predadores e parasitas (Nogueira Neto 1997). Este comportamento pode evitar também o parasitismo reprodutivo, onde rainhas não fecundadas buscam outros ninhos já estabelecidos para colocar seus ovos, aumentando com isso, seu sucesso evolutivo. Este comportamento ocorre principalmente à noite, período onde a maior parte das atividades está concentrada no interior da colônia (Oystaeyen et al. 2013). Como o tubo de acesso é fechado durante a noite em $N$. testaceicornis as chances de outras rainhas adentrarem nos ninhos diminuem.

O horário de abertura e fechamento variou entre as diferentes estações climáticas do ano. No período quente e úmido a abertura ocorreu em torno de 5 horas e 30 minutos, e o fechamento por volta das $19 \mathrm{~h}$ horas, ficando aberto por aproximadamente $13 \mathrm{~h}$ e $30 \mathrm{~min}$. No período frio e seco a abertura teve início por voltada das 7 horas e 20 minutos e terminou às 18 horas e 20 minutos, ficando aberto por aproximadamente 11 horas.

O tempo médio gasto pelas abelhas durante a abertura e fechamento do tubo foi respectivamente $82 \pm 30$ minutos e 149 \pm 53 minutos (Tabela 1). Comparando estes dois resultados pelo teste do qui-quadrado, podemos observar que há uma diferença significativa entre esses dois processos $\left(\chi^{2}=16,88\right.$; $p<0,0001$, teste do qui-quadrado). O tempo gasto durante o fechamento foi significativamente mais longo durante o período quente e úmido $\left(\chi^{2}=7,50 ; \mathrm{p}<0,0062\right)$, do que durante o período frio e seco. O tempo de fechamento do tubo mais longo no período quente e úmido mais rápido durante o período frio foi regulado pelo ritmo de atividade externa das abelhas, que são influenciadas pelos fatores climáticos (BIESMEIJER \& VRIES 2001; Silva et al. 2013). Assim, nos dias frios as abelhas retornam do campo mais cedo e fecham rapidamente a entrada do ninho para manter as condições do ninho ideais. Diferentemente, nas estações quentes do ano caracterizado por altas temperaturas e longos períodos de luminosidade, abelhas podem adiar o retorno ao ninho até depois do por do sol, comportamento que permite a entrada das últimas operárias que retornam do campo e por isso, e fechamento é mais lento (Michener 1974). Estudos com vespas nesta mesma localidade realizados por (Elisei et al. 2013), mostrou que as atividades de forrageamento são influenciadas principalmente pela velocidade do vento, temperatura e luminosidade e que na estação quente os fatores citados acima estão em condições ideais para o forrageamento deste grupo ao longo do dia, fato que pode estender o horário de atividade até o início da noite, o que parece está acontecendo com as abelhas no presente estudo. CASTRO 
(2009), estudando a atividade de voo em diferentes colônias de $N$. testaceicornis, na mesma área do presente trabalho, observouse que o período de atividade foi maior durante o período quente e úmido ( 7 horas e 18 horas), quando comparamos os dados com o período frio e seco ( 9 horas e 18 horas).

Os resultados demonstram que o processo de abertura e fechamento do tubo de acesso de $N$. testaceicornis é fundamental na regulação das condições internas da colônia e que esse processo é influenciado, principalmente, pelos fatores externos. Por isso, sofre alterações durante os diferentes períodos do ano. Desta forma, este trabalho fornece importantes informações sobre a biologia desta espécie, que podem ser empregadas em seu manejo, aumentar sua utilização em atividades comerciais e diminuir a perda de operárias durante a migração das colônias.
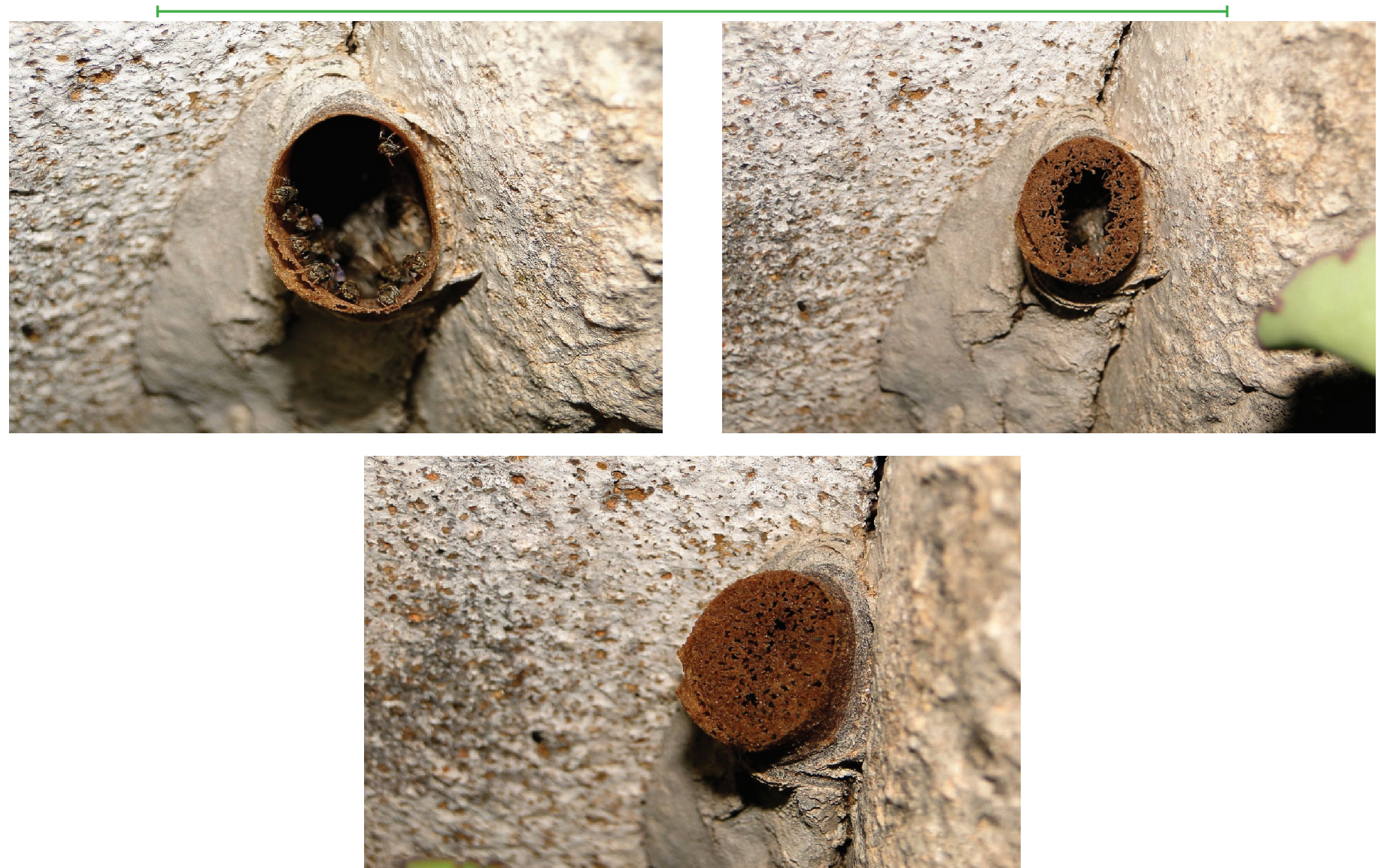

Figura 1. Sequência de fechamento do tubo de entrada de Nannotrigona testaceicornis, em uma área antrópica de Juiz de Fora, Minas Gerais, sudeste do Brasil.

\section{AGRADECIMENTOS}

Agradecemos a Mateus Farjado de Freitas S. Detoni pela ajuda na elaboração final deste trabalho.

\section{REFERÊNCIAS}

Biesmeijer, J.C. \& H. de Vries, 2001. Exploration and exploitation of food sources by social insect colonies: a revision of the scout-recruit concept. Behavioral Ecology and Sociobiology, 49: 89-99.

Biesmeijer, J.C., M. Giurfa, D. Koedam, S.G. Potts, D.M. Joel \& A. Dafni, 2005. Convergent evolution: floral guides, stingless bee nest entrances, and insectivorous pitchers. Naturwissenschaften, 92: 444-450.

Camargo, J.M.F \& Wittmann, D., 1989. Nest architecture and distribution of the primitive stingless bee, Mourella caerulea (Hymenoptera, Apidae, Meliponinae): evidence of the origin of Plebeia (s. lat.) on the Gondwana continent. Studies on Neotropical Fauna and Environment, 4, 213-229.

Camargo, J.M.F. \& S.R.M. Pedro, 2003. Meliponini neotropicais: o gênero Partamona Schwarz, 1939 (Hymenoptera, Apidae, Meliponini) - bionomia e biogeografia. Revista Brasileira de Entomologia. 47: 311-372.

Castro, L.C., 2009. Abelhas eusociais (Hymenoptera, Apidae) que ocorrem em jardins urbanos em Juiz de Fora, MG: recursos florais e atividade de vôo. (Dissertação de Mestrado). Universidade Federal de Juiz de Fora, Minas Gerais State, Brasil. 59p.
Couvillon, M.J., T. Wenseleers, V. Imperatriz-Fonseca, P. Nogueira-Neto \& F.L.W. Ratnieks, 2008. Comparative study in stingless bees (Meliponini) demonstrates that nest entrance size predicts traffic and defensivity. Journal of Evolutionary Biology, 21: 194-201.

Elisei, T., J. Nunes, C. Ribeiro Junior, A. Fernandes Junior \& F. Prezoto, 2013. What is the Ideal Weather for Social Wasp Polistes versicolor (Olivier) go to Forage? EntomoBrasilis, 6: 214-216.

Köppen, W., 1970. Roteiro para classificação climática. 6 p. [Mimeo]

Lindauer, M. \& W.E. Kerr, 1960. Communication between the workers of stingless bees. Bee World, 41: 29-41.

Michener, C.D., 1974. The Social Behavior of the Bees. Massachussets, Cambridge University. Ed. Havard University. 404p.

Michener, C.D., 2000. The Bees of the World. Johns Hopkins University Press, Baltimore, MD. 913p.

Nogueira Neto, P., 1997. Life and creation of stingless bees. Ed. Nogueirapis. São Paulo, SP, 445p.

Roubik, D.W., 1989. Ecology and Natural History of Tropical Bees. Cambridge University Press. Cambridge, New York, Port Chester, Melbourne, Sydney. 514p.

Silva, K.N., J.C.S., M. Dutra \& L.P. Polatto, 2013. Influência dos Fatores Ambientais e da Quantidade de Néctar na Atividade de Forrageio de Abelhas em Flores de Adenocalymma bracteatum (Cham.) DC. (Bignoniaceae). EntomoBrasilis, 6: 193-201. 
Silveira, F. A., G.A.R. Melo \& E.A.B. Almeida, 2002. Abelhas Brasileiras: Sistemática e Identificação. Belo Horizonte, Fernando A. Silveira. 253 p.

Slaa, E.J., L.A. Sanchez, M. Sandi \&W. Salazar, 2000. A scientific note on the use of stingless bees for commercial pollination in enclosure. Apidologie 31: 141-142.

Oystaeyen, A.V., D.A. Alves, R.C. Oliveira, D.L. do Nascimento, F.S. do Nascimento, J. Billen, 2013. Sneaky queens in Melipona bees selectively detect and infiltrate queenless colonies. Animal Behaviour, 86: 603-609.

Wille, A. \& C.D. Michener, 1973. The Nest Architecture of Stingless Bees with Special Reference to Those of Costa Rica. Revista de Biologia Tropical, 1: 1-278.
Wilson, E.O., 1971. The Insect Societies. The belknap Press of Harvard University, Press Cambridge, Massachussets and London, England. 548p.

\section{Recebido em: 09/07/2014}

Aceito em: 01/11/2014

\section{Como citar este artigo:}

Alves, L.H, L. A. Sousa, A.F. Barreto-Lima, G.M.F. Mucci \& F. Prezoto, 2015. Biologia de Abertura e Fechamento do Tubo de Acesso de Nannotrigona testaceicornis (Lepeletier) (Apidae: Meliponini). EntomoBrasilis, 8 (1): 08-11.

Acessível em: $\underline{\text { doi:10.12741/ebrasilis.v8i1.458 }}$
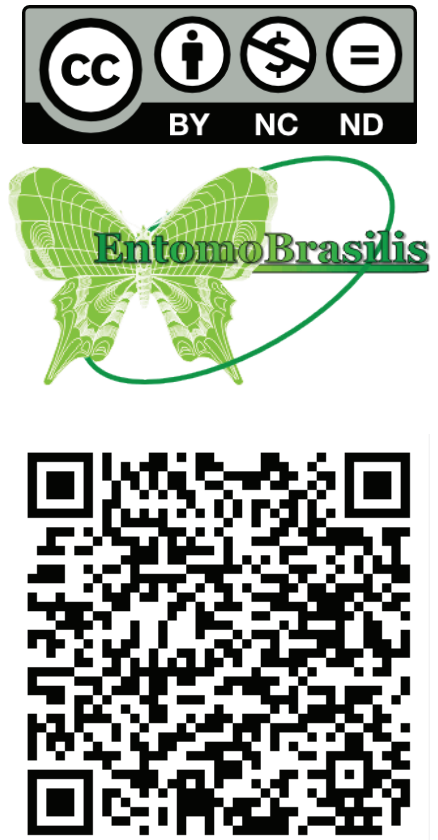